\title{
]jfis
}

\section{New Method of Internal Type-2 Fuzzy-Based CNN for Image Classification}

\author{
P. Murugeswari ${ }^{1}$ and S. Vijayalakshmi ${ }^{2}$ \\ ${ }^{1}$ Department of Computer Science and Engineering, Karpagam College of Engineering, \\ Coimbatore,Tamilnadu, India \\ ${ }^{2}$ Department of Computer Applications, NMS S. Vellaichamy Nadar College, Madurai, Tamilnadu, India
}

\begin{abstract}
In the last two decades, neural networks and fuzzy logic have been successfully implemented in intelligent systems. The fuzzy neural network (FNN) system framework infers the union of fuzzy logic and neural network system framework thoughts, which consolidates their advantages. The FNN system is applied in several scientific and engineering areas. Wherever there is uncertainty associated with the data, fuzzy logic places a vital rule. The fuzzy set can effectively represent and handle uncertain information. The main objective of the FNN system is to achieve a high level of accuracy by including the fuzzy logic in either the neural network structures, activation functions, or learning algorithms. In computer vision and intelligent systems, convolutional neural networks (CNNs) have more popular architectures, and their performance is excellent in many applications. In this paper, fuzzy-based CNN image classification methods are analyzed, and an interval type- 2 fuzzy-based CNN is proposed. The experimental results indicated that the performance of the proposed method was good.
\end{abstract}

Keywords: CNN, FCNN, Fuzzy logic, Interval type-2 fuzzy logic, Feature extraction, Computer vision, Image classification

\section{Introduction}

In computer vision image classification, a given image is characterized into a pre-defined characterized class. Customary image classification includes feature extraction and classification modules. The feature extraction includes extracting a superior degree of pixel information from raw pixels, which will capture the greatness among the classifications. Normally, this process is performed in an unsupervised manner wherein the classes of the picture have nothing to do with the data extracted from pixels. Few typical and generally utilized feature extractions are GIST, HOG, SIFT, and LBP. After feature extraction, a classification module is prepared with the image and its related names. Few tests for this module include SVM, logistic regression, random forest, and choice trees.

Recurrent neural networks (RNNs), long short-term memory (LSTM), artificial neural networks (ANNs), and convolutional neural networks (CNNs) are the most popular neural network architectures. A CNN is suitable for image databases, and it works amazingly in computer vision tasks, such as image classification [1, 2], object detection [3], and image recognition [4]. It does not include an explicit element extractor. It extracts and characterizes modules in a coordinated framework and determines ways to separate representations from the pictures and orders them depending on regulated information. 
A CNN is utilized in various assignments that have an extraordinary presentation in various applications. CNNs have been presenting an employable class of models for better information on image details, achieving better image acknowledgment, segmentation, identification, and retrieval. CNN structures are productively and successfully utilized in several pattern and image recognition applications [5], for example, motion acknowledgment [4, 6], face acknowledgment [7, 8], object characterization [9, 10], and scene description creation [11].

Zadeh [12] presented the idea of fuzzy logic (type-1 fuzzy) for tackling control framework-related issues. Later, analysts have contributed several fascinating applications to the field of computer vision. Notably, a type-2 fuzzy set (T2FS) was presented by Zadeh [13] in 1975, and it was further created by Jerry M. Mendel. In a type-1 fuzzy set (T1FS), the degree of participation is determined by a crisp number in the interval $[1,0]$. In T2FS, the degree of participation is itself fuzzy and is indicated by secondary membership functions (MFs). If the secondary MF values are at their limit of 1 at each point, which is called an interval type-2 fuzzy set (IT2FS) [13-15], the T2FS incorporates a third measurement and an impression of uncertainty, as depicted in Figure 1, which gives an additional level of opportunity to deal with uncertainty. This additional level of fuzziness provides an increasingly capable method to deal with uncertainty. Figure 2 illustrates the secondary MFs (third element) of the T1FS (Figure 2(a)), IT2FS (Figure 2(b)), and general T2FS (Figure 2(c)), as initiated by an information p, similar to that shown in Figure 1.

Particularly, type-1 FCM has become the most notable calculation used in group investigations. Numerous analysts have demonstrated that there are imperatives in the limitations of T1FSs to show and break the effect of uncertainties, because

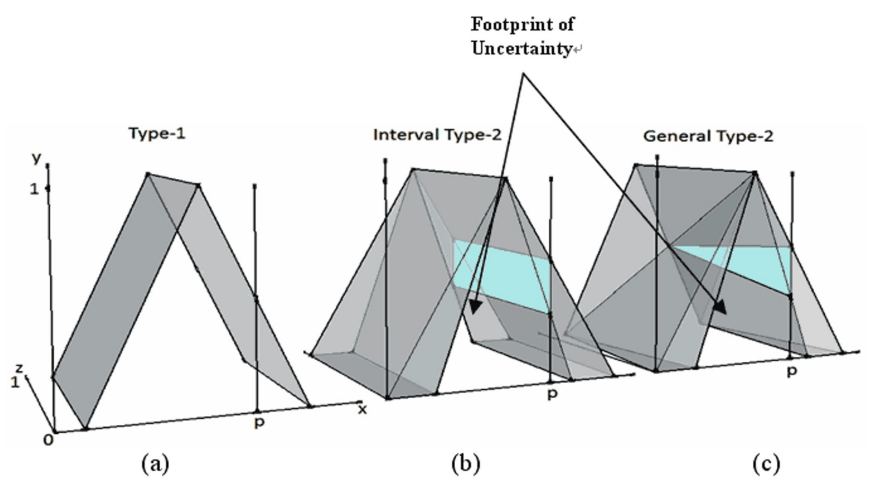

Figure 1. Case of three kinds of fuzzy sets. A similar information $p$ is applied to each fuzzy set. (a) T1FS, (b) IT2FS, and (c) T2FS.

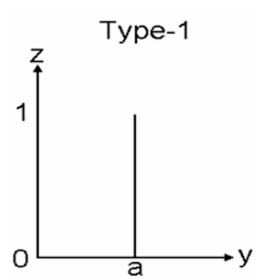

(a)

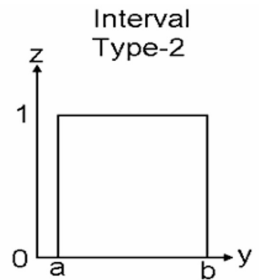

(b)

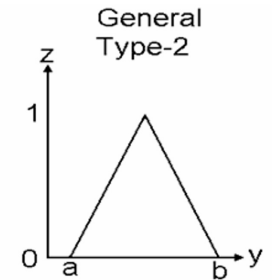

(c)
Figure 2. Perspective on the secondary membership functions (three dimensions) initiated by an information $\mathrm{p}$ for (a) T1FS, (b) IT2FS, and (c) T2FS.

their interest grades are crisp. A T2FS is spoken to by MFs that are themselves fuzzy. An IT2FS [16], an exceptional instance of T2FS, is currently the one most generally utilized, considering its decreased computational expense. An IT2FS is restricted by two T1FSs, one above and one below, that are called upper MF (UMF) and lower MF (LMF), and the domain among the UMF and LMF is called the footprint of uncertainty (FOU). Thus, T2FS exhibits various uncertainties; however, the computational unpredictability increases owing to its extra component of optional evaluations of every essential enrolment. Certain model applications include type-2 fuzzy clustering [17], Gaussian noise filter, classification of coded video streams, medical applications, and color picture division.

Recently, fuzzy logic and neural networks have been widely applied to solve real-world problems. Fuzzy logic is a set of mathematical principles used for knowledge representation, based on the degrees of membership, as opposed to the classical binary logic. It is a powerful tool to tackle imprecision and uncertainty and was initially introduced to provide robust and low-cost solutions for real-world problems. Generally, type-1 fuzzy logic systems (T1FLS) have been implemented in several systems such as forecasting systems, control systems, databases, and healthcare clinical diagnoses.

The drawback of the conventional type-1 fuzzy logic system is its limited capability to handle data uncertainties directly, as certain designed systems face high level of uncertainties that can affect the performance of the systems. The type- 2 fuzzy logic system (T2FLS) is an extension of the former with the intention of being able to model the uncertainties that invariably exist in the rule base because the MFs of type-2 fuzzy systems are themselves fuzzy. It provides a powerful framework for representing and handling such types of uncertainties. An interval type-2 fuzzy logic system (IT2FLS), which is a special case of T2FLS, has been applied to solve real-world problems. Recent theoretical and practical studies confirm that IT2FLSs 
adequately handle uncertainties compared to that by T1FLSs, and an increasing number of applications of IT2FLSs is expected in different fields of science and engineering. T1FLSs and IT2FLSs have been applied in a wide variety of areas to solve problems.

\section{Literature Survey}

The CNN is a type of neural network that has indicated commendable execution when faced with several challenges related to computer vision and image processing. A part of the invigorating application areas of CNN fuse image classification and segmentation [18], object detection [3], video processing [19], natural language processing [20, 21], and speech recognition [22, 23]. The learning limit of a significant CNN is basically a result of the usage of various component extractions composes that can normally take in exposé from the data. The availability of abundant data and improvement in the gear development has accelerated the investigation in CNNs, and starting late attractive profound $\mathrm{CNN}$ models have been represented. A few moving plans for advancement of CNNs have been investigated [7], for example, the utilization of various activation and loss functions, parameter streamlining, regularization, and compositional advancements.

Karnik et al. [24] stated that the use of a T1FS to model a word is scientifically incorrect because a word is uncertain and a T1FS is certain. Therefore, he conducted in-depth research on type-2 fuzzy and contributed several papers [24-26] on type-2 fuzzy logic. Based on that, several researchers have contributed several algorithms for their applications. For example, the classification of coded video streams, diagnosis of diseases, pre-processing radiographic images, medical image applications, transport scheduling, forecasting of time series, learning linguistic membership grades, inference engine design, and control of mobile robots. The computational complexity is high in type-2 fuzzy. Therefore, the type-2 fuzzy set is simplified into an IT2 Fuzzy, in which the computational complexity can be significantly reduced in appropriate applications.

Recently, fuzzy logic and neural networks have been widely applied to solve real-world problems. Fuzzy logic includes several mathematical standards for information representation depending on degrees of participation, as opposed to the binary logic. It is an incredible asset to handle imprecision and uncertainty, and it was introduced to provide robust and low-cost resolution for real-world problems [27]. Particularly, type-1 fuzzy logic frameworks have been executed in numerous sys- tems to a wider scale, including approximation and forecasting systems, control systems, databases, and healthcare clinical diagnosis.

Researchers have successfully combined and implemented neural networks and fuzzy logic in intelligent systems. The fuzzy neural network (FNN) system framework implies the combination of fuzzy logic and neural network system ideas, which incorporates their benefits. This FNN is applied in several scientific and engineering areas, such as text sentient evaluation [28], object classification with small training database [3], emotion features extraction from text [29], comprehension of emotions in movies [30], real world objects and image classification [19, 31], Marathi handwritten numerals recognition [32, 33], traffic flow prediction [34], electric load prediction [35], and handwritten digits recognition [36]. Keller and Hunt [37] proposed hierarchical deep neural network fuzzy systems that obtain information from both fuzzy and neural representations. Price et al. [38] proposed the introduction of the fuzzy layers for deep learning, experiencing the choice of different combination procedures and total yields from bestin-class pre-prepared models, for example, AlexNet, VGG16, GoogLeNet, Inception-v3, and ResNet-18.

\section{Comparison of FCNN Architectures}

Generally, CNN architectures include two phases: feature extraction and classification. The FCNN is a combination of CNN and fuzzy logic; therefore, the fuzzy logic may include either a feature extraction phase or a classification phase. Depending on the application, researchers have proposed various FCNN architectures, including fuzzy logic in the feature extraction phase or classification phase. Here, the two FCNN architectures, with fuzzy logic included in the classification phase, were compared for image classification. Hsu et al. [3] have integrated a CNN with a fuzzy neural network (FCNN model 1), where the FNN summarizes the feature information from every fuzzy map. Korshunova [10] (FCNN model 2) proposed a CFNN architecture that includes a fuzzy layer, which is situated between the convolutional network and classifier.

\section{Interval Type-2 Fuzzy CNN}

The new IT2FCNN architecture integrates the features of the CNN and FNN. It integrates the interval type-2 fuzzy rectification unit (IT2FRU) [39] activation function in convolution for feature extraction in $\mathrm{CNN}$ and interval type-2 fuzzy-based 


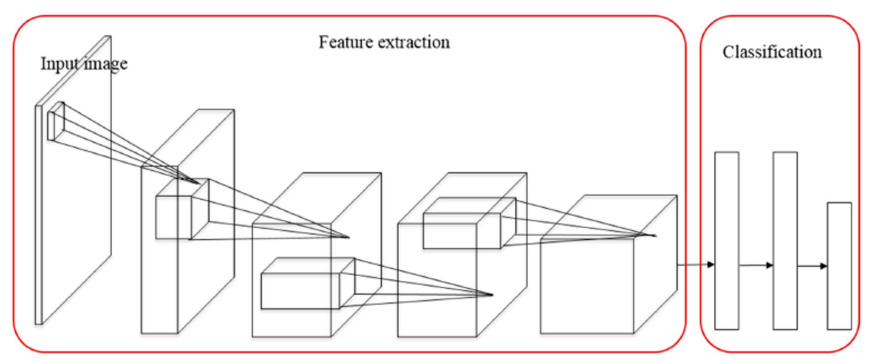

Figure 3. Structure of a convolutional neural network $(\mathrm{CNN})$.

classification in the fuzzy layer. This method combines the advantages of both network architectures and interval type-2 fuzzy logic. The IT2FCNN architecture includes four types of layers: i) convolutional layer with IT2FRU, ii) pooling layer, iii) fuzzy layer, and iv) fuzzy classifier.

The convolutional neural framework obtains a data image and performs the course of action of convolutional and pooling layers. The fuzzy layer performs grouping using the interval type-2 fuzzy clustering algorithm. The yields of the fuzzy layer neurons represent the estimations of the participation capacities for the fuzzy clustering of input data. The information point cluster is chosen based on their participation grade. These characteristics demonstrate the promise of a classifier. Its yield is the full IT2FCNN yield, which is the class score for the picture. Leave $\mathrm{C}$ alone the number of neurons in the fuzzy layer (the quantity of clusters). The neurons of the fuzzy layer commencement limits are IT2FRUs showing the interest of the information vector $\mathrm{x}$ to all $\mathrm{L}$ groups.

IT2FRU employs the following equalities: $Z=0$, to guarantee that $\sigma=0 \Rightarrow \phi_{o}=0$. Additionally, the height of the LMFs is employed as $m 2=\alpha, m 1=m 3=1-\alpha$, as suggested in [24]. The resulting IT2FM $(\phi 0(\sigma))$ for $\sigma \in[0,1]$ can be formulated as follows:

$$
\phi_{o}(\sigma)=\operatorname{P\sigma k}(\sigma)
$$

where $k(\sigma)$ is defined as

$$
k(\sigma)=\frac{1}{2}\left(\frac{1}{\alpha+\sigma-\alpha \sigma}+\frac{-1+\alpha}{-1+\alpha \sigma}\right) .
$$

Similarly, for the input interval $\sigma \in[-1,0]$ the IT2FM can be derived as follows:

$$
\phi_{o}(\sigma)=N \sigma k(-\sigma)
$$

The activation unit can be formulated by arranging Eqs. (1) and (3) as follows:

$$
f(\sigma)=\left\{\begin{array}{l}
P \sigma k(\sigma), \text { if } \sigma>0, \\
N \sigma k(-\sigma), \text { if } \sigma \leq 0 .
\end{array}\right.
$$

The parameter $P$ controls the incline of the capacity in the positive quadrant, whereas the parameter $N$ controls the slant of the capacity in the negative quadrant. The resulting output of the IT2FRU can be a linear or nonlinear activation depending on the selection of the parameters. The IT2FRU has three learnable parameters $P, N$, and $\alpha$.

The vector $x=\left[x_{1}, x_{2}, \ldots, x_{j}, \ldots, x_{n}\right]$ deals with the commitment of the framework, and the fuzzy layer forms a vector involving the degrees of having a spot $\mathrm{x}$ with specific cluster territories: $\left[v_{i} v_{2} \ldots v_{j}\right]$. The parts $\left(\overline{\mathrm{u}}_{\mathrm{j}}\left(\mathrm{x}_{\mathrm{i}}\right), \underline{\mathrm{u}}_{\mathrm{j}}\left(\mathrm{x}_{\mathrm{i}}\right)\right)$ are determined utilizing Eq. (5) to fulfill the standardization condition utilizing Eq. (6) for each preparation test vector $\mathrm{x}^{(\mathrm{k})}, \mathrm{k}=1$,

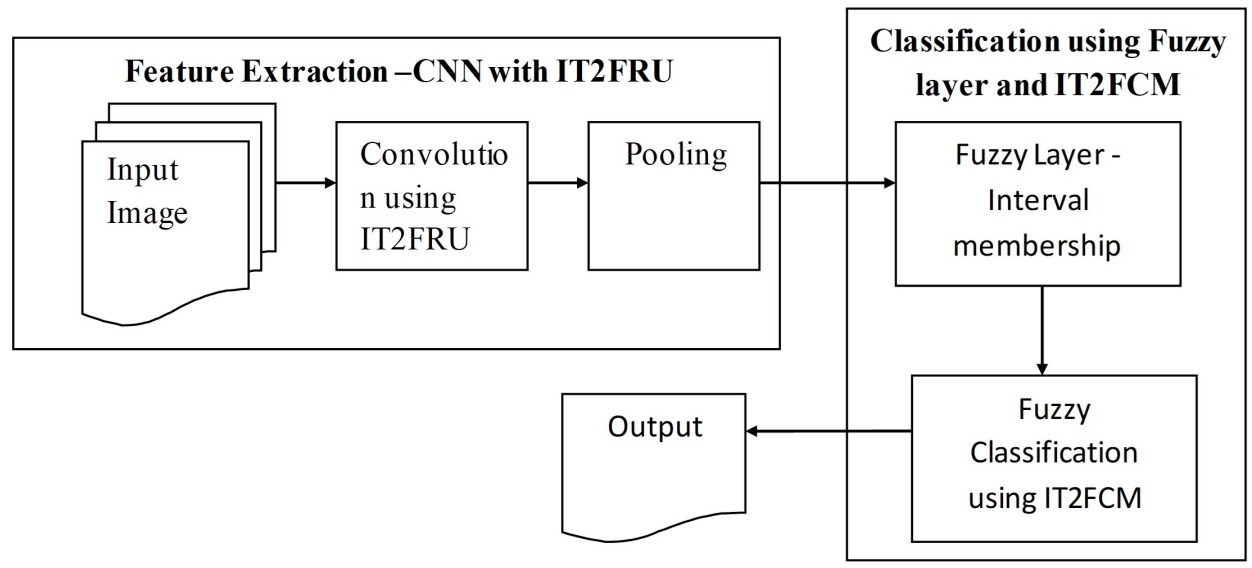

Figure 4. Outline of the proposed method IT2FCNN. 
..., $\mathrm{K}$, where $\mathrm{K}$ denotes the number of vectors in the ready set. The yields of neurons of the fuzzy layer are used as the commitments of the classifier.

$$
\begin{aligned}
& \widetilde{\mu}_{i}\left(x^{k}\right)=f \sum_{j=1}^{n} x_{j}^{k}, \\
& \sum_{i=1}^{L} \widetilde{\mu}_{i}\left(x^{k}\right)=1 .
\end{aligned}
$$

- The Interval Type-2 Fuzzy Membership becomes

$$
\bar{u}_{j}\left(x_{i}\right)=\left\{\begin{array}{l}
\frac{\sum_{k=1}^{C}\left(\left(d_{j i} / d_{k i}\right)+\alpha\left(d_{j i} / d_{k i}\right) \delta\right)^{2 /\left(m_{1}-1\right)}}{\sum_{k=1}^{C}\left(\left(d_{j i} / d_{k i}\right)+\alpha\left(d_{j i} / d_{k i}\right) \delta\right)^{2 /\left(m_{1}-1\right)}}, \\
\text { if } \frac{1}{\sum_{k=1}^{C}\left(d_{j i} / d_{k i}\right)}<\frac{1}{C}, \\
\frac{\sum_{k=1}^{C}\left(\left(d_{j i} / d_{k i}\right)+\alpha\left(d_{j i} / d_{k i}\right) \delta\right)^{2 /\left(m_{2}-1\right)}}{\sum_{k=1}^{C}\left(\left(d_{j i} / d_{k i}\right)+\alpha\left(d_{j i} / d_{k i}\right) \delta\right)^{2 /\left(m_{2}-1\right)}} \\
\text { otherwise, }
\end{array}\right.
$$

$$
\underline{u}_{j}\left(x_{i}\right)=\left\{\begin{array}{l}
\frac{\sum_{k=1}^{C}\left(\left(d_{j i} / d_{k i}\right)+\alpha\left(d_{j i} / d_{k i}\right) \delta\right)^{2 /\left(m_{1}-1\right)}}{\sum_{k=1}^{C}\left(\left(d_{j i} / d_{k i}\right)+\alpha\left(d_{j i} / d_{k i}\right) \delta\right)^{2 /\left(m_{1}-1\right)}} \\
\text { if } \frac{1}{\sum_{k=1}^{C}\left(d_{j i} / d_{k i}\right)} \geq \frac{1}{C}, \\
\frac{\sum_{k=1}^{C}\left(\left(d_{j i} / d_{k i}\right)+\alpha\left(d_{j i} / d_{k i}\right) \delta\right)^{2 /\left(m_{2}-1\right)}}{\sum_{k=1}^{C}\left(\left(d_{j i} / d_{k i}\right)+\alpha\left(d_{j i} / d_{k i}\right) \delta\right)^{2 /\left(m_{2}-1\right)}} \\
\text { otherwise }
\end{array}\right.
$$

- Updating cluster centers

$$
v_{j}=\frac{v_{L}+v_{R}}{2} .
$$

- Type reduction and hard-partitioning can be acquired as follows:

$$
\begin{aligned}
& \mathrm{u}_{\mathrm{j}}\left(\mathrm{x}_{\mathrm{i}}\right)=\frac{\mathrm{u}_{\mathrm{j}}^{\mathrm{R}}\left(\mathrm{x}_{\mathrm{i}}\right)+\mathrm{u}_{\mathrm{j}}^{\mathrm{L}}\left(\mathrm{x}_{\mathrm{i}}\right)}{2}, j=1, \ldots, C, \\
& u_{j}^{R}\left(x_{i}\right)=\frac{\sum_{l=1}^{M} u_{j l}\left(x_{i}\right)}{M},
\end{aligned}
$$

where

$$
u_{j l}\left(x_{i}\right)= \begin{cases}\bar{u}_{j}\left(x_{i}\right), & \text { if } x_{i l} \text { uses } \bar{u}_{j}\left(x_{i}\right) \text { for } v_{j}^{R} \\ \underline{u}_{j}\left(x_{i}\right), & \text { otherwise }\end{cases}
$$

and

$$
u_{j}^{L}\left(x_{i}\right)=\frac{\sum_{l=1}^{M} u_{j l}\left(x_{i}\right)}{M},
$$

where

$$
u_{j l}\left(x_{i}\right)= \begin{cases}\bar{u}_{j}\left(x_{i}\right), & \text { if } x_{i l} \text { uses } \bar{u}_{j}\left(x_{i}\right) \text { for } v_{j}^{L}, \\ \underline{u}_{j}\left(x_{i}\right), & \text { otherwise. }\end{cases}
$$

The processes of IT2FCNN are isolated into three phases: the information design (picture) undergoes a progression of changes; subsequently, a vector of significant level attributes is framed; further, the fuzzy layer executes a groundwork dissemination of the information into fuzzy groups; finally, the totally related layers execute the plan, consigning the result class name to each get-together of clusters.

\section{Experimental Result}

\subsection{Data set and Experimental setup}

Various datasets are available for the application of neural networks. The most popular datasets are CIFAR-10, Caltech101, and ImageNet. The CIFAR-10 dataset includes 60,000 images in 10 classes, with 6,000 images per class. The Caltech101 dataset includes 101 classes with 40 to 800 images per class. The ImageNet dataset includes more than 14 million images, with 20,000 categories. The experiments were executed in a Windows 764 piece working framework, and the principle memory and capacity limit of the PC frameworks were 8 GB RAM and 1 TB, respectively. An Intel(R) Core(TM) i5-4590 CPU @ 3.30GHz and an NVIDIA GeForce GT 705 graphics card were used. The product utilized in this investigation were Python 3.6 and Matlab. The PyCharm IDE was utilized and organized by utilizing the Keras libraries on the PyCharm exploratory stage.

\subsection{Training the Architecture}

The training of IT2FCNN was the foremost step that included three autonomous steps of the three components of the network. First, the model was prepared utilizing the theoretical properties of the input image by the back propagation model. In the second part of the model, the fuzzy layer was tuned using the competitive learning scheme, which implied choosing the parameters of the MF for setting the cluster centers. Various fuzzy clustering algorithms are available. Here, IT2FCM was used for clustering. Finally, the classifier was trained using the weights 
Table 1. Performance comparison analysis with various fine tuning epochs $(3,5$, and 7$)$

\begin{tabular}{|c|c|c|c|c|c|c|c|c|c|c|c|c|c|}
\hline Model & $\begin{array}{c}\text { Fine } \\
\text { tuning } \\
\text { epochs }\end{array}$ & \multicolumn{4}{|c|}{ Dog Vs Cat } & \multicolumn{4}{|c|}{ Lion Vs Tiger } & \multicolumn{4}{|c|}{ Horse Vs Donkey } \\
\hline & 5 & 51 & 60 & 61 & 65 & 61 & 64 & 65 & 68 & 60 & 62 & 64 & 68 \\
\hline & 7 & 54 & 65 & 68 & 72 & 66 & 68 & 72 & 74 & 65 & 68 & 71 & 74 \\
\hline & 5 & 53 & 62 & 61 & 65 & 62 & 63 & 65 & 68 & 62 & 64 & 68 & 70 \\
\hline & 7 & 54 & 64 & 67 & 73 & 68 & 69 & 73 & 76 & 64 & 69 & 72 & 76 \\
\hline \multirow[t]{3}{*}{ GoogLeNet } & 3 & 42 & 56 & 57 & 61 & 58 & 61 & 61 & 64 & 56 & 61 & 64 & 64 \\
\hline & 5 & 54 & 61 & 64 & 68 & 64 & 68 & 68 & 72 & 61 & 68 & 70 & 73 \\
\hline & 7 & 57 & 68 & 70 & 74 & 68 & 70 & 74 & 78 & 68 & 70 & 74 & 78 \\
\hline & 7 & 55 & 70 & 72 & 76 & 70 & 72 & 76 & 78 & 70 & 72 & 74 & 78 \\
\hline \multirow[t]{3}{*}{ ResNet50 } & 3 & 43 & 56 & 56 & 63 & 59 & 61 & 63 & 65 & 56 & 61 & 64 & 65 \\
\hline & 5 & 54 & 61 & 62 & 67 & 63 & 62 & 67 & 69 & 61 & 62 & 65 & 69 \\
\hline & 7 & 56 & 69 & 71 & 78 & 68 & 72 & 76 & 79 & 69 & 72 & 74 & 79 \\
\hline
\end{tabular}

tuning in the fully connected layers. After the completion of training, the IT2FCNN was ready for implementation. The image pixel cluster was taken care of by the CFNN. The yield of the system was input image $\mathrm{p}$ class scores, and the image was allocated to the class max score esteem class.

\subsection{The comparative Analysis}

In this study, AlexNet, ZFNet, GoogLeNet, VGGNet16, and ResNet50 pretrained on CIFAR, ImageNet, and Caltech101 datasets were chosen for the experiment. The CFNN model finetuned AlexNet, ZFNet, GoogLeNet, VGGNet16, and ResNet50 to classify the images. Here, 3, 5, and 7 epochs were used for training the models. In the fuzzy layer, IT2FCM clustering was used to cluster the set of data several times with different numbers of clusters. When the fuzzy partition coefficient was maximized, the number of clusters equal to that number was chosen for the experiment. Adam, the stochastic optimization method, was used for classifier training (to tune weights) for the fully connected layer.

Table 1 presents the model performance comparison between the existing CNN and fuzzy-based CNN architectures. Figure 5 clearly shows that the fuzzy-based CNN architecture increases the performance accuracy compared to the traditional $\mathrm{CNN}$ architecture. The investigation distinctly indicated that remembering the fuzzy layer for the $\mathrm{CNN}$ gave a high caliber of exactness compared to a customary CNN.

\subsection{The Comparison of RMSE, MSE, and MAE}

The percentage error (\% error), mean squared error (MSE), root mean squared error (RMSE), and mean absolute percentage error (MAPE) are the performance criteria for image classification. The corresponding calculation methods are defined as follows:

$$
\begin{aligned}
& \% \operatorname{Errr}=\frac{\left|\mathrm{y}_{\mathrm{i}}-\overline{\mathrm{y}}_{\mathrm{i}}\right|}{\mathrm{y}_{\mathrm{i}}} \times 100, \\
& M S E=\frac{1}{N} \sum_{i=1}^{N}\left(y^{x}-y\right)^{2}, \\
& R M S E=\sqrt{\frac{1}{N} \sum_{i=1}^{N}\left(y^{x}-y\right)^{2},} \\
& M A P E=\frac{100}{N} x \int_{i=1}^{N}\left|\frac{y-y^{x}}{y}\right| .
\end{aligned}
$$

Table 2 presents the comparison of FCNN models with IT2FCNN based on MSE and RMSE. The results indicated that the proposed method produced a good result. 


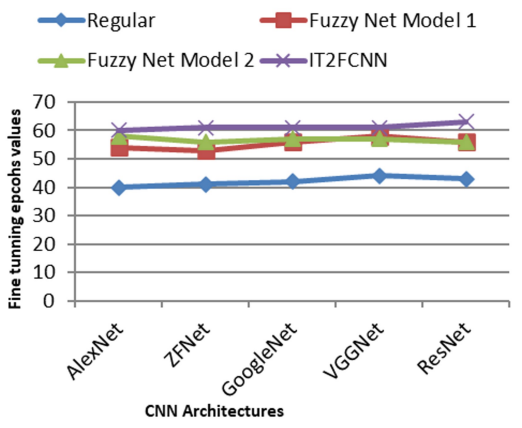

(a)

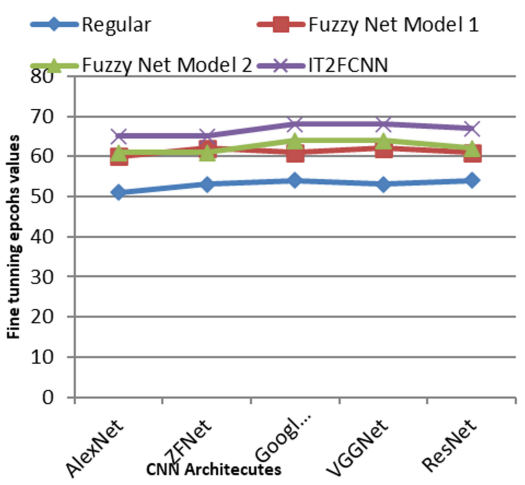

(b)

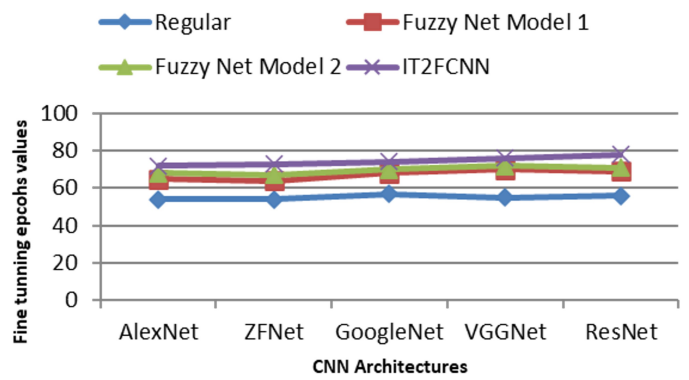

(c)

Figure 5. Performance comparison analysis for Dog vs. Cat with fine tuning epochs 3 (a), fine tuning epochs 5 (b), and fine tuning epochs 7 (c).

\section{Conclusions}

The exploratory outcomes indicate that fuzzy- neural networks represent a ground-breaking and reasonable option in contrast to regular arrangement strategies. The merging of fuzzy logic with neural network applications is progressively expert in decision marking systems. In the proposed method, the CNN was used to extract the features and integrate the interval type-2 fuzzy to classify the images, which increased the accuracy of the experiment. In addition, our test results demonstrated that it was conceivable to improve testing exactness by watching the conveyance of pixels in include maps and modifying the mem-
Table 2. Comparison of FCNN models with IT2FCNN based on MSE and RMSE

\begin{tabular}{lccc}
\hline Models & MSE & RMSE & MAPE \\
\hline AlexNet & & & \\
FCNN Model 1 & .00245 & .052 & 4.4 \\
FCNN Model 2 & .00183 & .043 & 3.2 \\
IT2FCNN & .00123 & .035 & 2.4 \\
ZFNet & & & \\
FCNN Model 1 & .00254 & .054 & 4.2 \\
FCNN Model 2 & .00143 & .045 & 3.1 \\
IT2FCNN & .00134 & .037 & 2.1 \\
GoogLeNet & & & \\
FCNN Model 1 & .00249 & .053 & 4.2 \\
FCNN Model 2 & .00197 & .041 & 3.2 \\
IT2FCNN & .00123 & .036 & 2.0 \\
VGGNet16 & & & \\
FCNN Model 1 & .00244 & .056 & 4.2 \\
FCNN Model 2 & .00158 & .047 & 3.0 \\
IT2FCNN & .00198 & .039 & 2.2 \\
ResNet50 & & & \\
FCNN Model 1 & .00268 & .058 & 4.1 \\
FCNN Model 2 & .00139 & .046 & 3.1 \\
IT2FCNN & .00132 & .037 & 2.1 \\
\hline
\end{tabular}

bership function. This method provided a better solution and had more advantages than the other existing methods. Although the results are more optimistic, image classification based on interval type-2 fuzzy logic still requires further research.

\section{Conflict of Interest}

No potential conflict of interest relevant to this article was reported.

\section{References}

[1] A. G. Howard, "Some improvements on deep convolutional neural network based image classification," in Proceedings of the 2nd International Conference on Learning Representations, Banff, Canada, 2014.

[2] N. Jmour, S. Zayen, and A. Abdelkrim, "Convolutional neural networks for image classification," in Proceedings of 2018 International Conference on Advanced Systems and Electric Technologies (IC ASET), Hammamet, 
Tunisia, 2018, pp. 397-402. https://doi.org/10.1109/ASET. 2018.8379889

[3] M. J. Hsu, Y. H. Chien, W. Y. Wang, and C. C. Hsu, "A convolutional fuzzy neural network architecture for object classification with small training database," International Journal of Fuzzy Systems, vol. 22, no. 1, pp. 1-10, 2020. https://doi.org/10.1007/s40815-019-00764-1

[4] X. Y. Wu, "A hand gesture recognition algorithm based on DC-CNN," Multimedia Tools and Applications, vol. 79, pp. 9193-9205, 2020. https://doi.org/10.1007/s11042-0197193-4

[5] L. Li, S. Qin, Z. Lu, K. Xu, and Z. Hu, "One-shot learning gesture recognition based on joint training of 3D ResNet and memory module," Multimedia Tools and Applications, vol. 79, no. 9, pp. 6727-6757, 2020. https://doi.org/10. 1007/s11042-019-08429-9

[6] Z. Zeng, Q. Gong, and J. Zhang, "CNN model design of gesture recognition based on TensorFlow framework," in Proceedings of 2019 IEEE 3rd Information Technology, Networking, Electronic and Automation Control Conference (ITNEC), Chengdu, China, 2019, pp. 1062-1067. https://doi.org/10.1109/ITNEC.2019.8729185

[7] C. J. Lin, C. H. Lin, C. C. Sun, and S. H. Wang, "Evolutionary-fuzzy-integral-based convolutional neural networks for facial image classification," Electronics, vol. 8, no. 9, article no. 997, 2019. https://doi.org/10.3390/ electronics 8090997

[8] T. Ahonen, A. Hadid, and M. Pietikainen, "Face description with local binary patterns: application to face recognition," IEEE Transactions on Pattern Analysis and Machine Intelligence, vol. 28, no. 12, pp. 2037-2041, 2006. https://doi.org/10.1109/TPAMI.2006.244

[9] W. Wang, Y. Yang, X. Wang, W. Wang, and J. Li, "Development of convolutional neural network and its application in image classification: a survey," Optical Engineering, vol. 58, no. 4, article no. 040901, 2019. https: //doi.org/10.1117/1.OE.58.4.040901

[10] K. P. Korshunova, "A convolutional fuzzy neural network for image classification," in Proceedings of 2018 3rd Russian-Pacific Conference on Computer Technology and Applications (RPC), Vladivostok, Russia, 2018, pp. 1-4. https://doi.org/10.1109/RPC.2018.8482211
[11] R. Chauhan, K. K. Ghanshala, and R. C. Joshi, “Convolutional neural network (CNN) for image detection and recognition," in Proceedings of 2018 1st International Conference on Secure Cyber Computing and Communication (ICSCCC), Jalandhar, India, 2018, pp. 278-282. https://doi.org/10.1109/ICSCCC.2018.8703316

[12] L. A. Zadeh, "Fuzzy sets," Information and Control, vol. 8, no. 3, pp. 338-353, 1965. https://doi.org/10.1016/S00199958(65)90241-X

[13] L. A. Zadeh, "The concept of a linguistic variable and its application to approximate reasoning-III," Information Sciences, vol. 8, no. 3, pp. 199-249, 1975. https://doi.org/ 10.1016/0020-0255(75)90017-1

[14] J. M. Mendel, R. I. John, and F. Liu, "Interval type-2 fuzzy logic systems made simple," IEEE Transactions on Fuzzy Systems, vol. 14, no. 6, pp. 808-821, 2006. https: //doi.org/10.1109/TFUZZ.2006.879986

[15] J. Zeng and Z. Q. Liu, "Type-2 fuzzy sets for handling uncertainty in pattern recognition," in Proceedings of 2006 IEEE International Conference on Fuzzy Systems, Vancouver, Canada, 2006, pp. 1247-1252. https: //doi.org/10.1109/FUZZY.2006.1681869

[16] C. Hwang and F. C. H. Rhee, "Uncertain fuzzy clustering: Interval type-2 fuzzy approach to C-means," IEEE Transactions on Fuzzy Systems, vol. 15, no. 1, pp. 107-120, 2007. https://doi.org/10.1109/TFUZZ.2006.889763

[17] N. N. Karnik and J. M. Mendel, "Applications of type2 fuzzy logic systems to forecasting of time-series," Information Sciences, vol. 120, no. 1-4, pp. 89-111, 1999. https://doi.org/10.1016/S0020-0255(99)00067-5

[18] A. D. Kulkarni and C. D. Cavanaugh, "Fuzzy neural network models for classification," Applied Intelligence, vol. 12, no. 3, pp. 207-215, 2000. https://doi.org/10.1023/A: 1008367007808

[19] T. Guo, J. Dong, H. Li, and Y. Gao, "Simple convolutional neural network on image classification," in Proceedings of 2017 IEEE 2nd International Conference on Big Data Analysis (ICBDA), Beijing, China, 2017, pp. 721-724. https://doi.org/10.1109/ICBDA.2017.8078730

[20] E. Cambria and B. White, "Jumping NLP curves: a review of natural language processing research," IEEE Compu- 
tational Intelligence Magazine, vol. 9, no. 2, pp. 48-57, 2014. https://doi.org/10.1109/MCI.2014.2307227

[21] W. Wang and J. Gang, “Application of convolutional neural network in natural language processing," in Proceedings of 2018 International Conference on Information Systems and Computer Aided Education (ICISCAE), Changchun, China, 2018, pp. 64-70. https://doi.org/10. 1109/ICISCAE.2018.8666928

[22] M. A. Haque, A. Verma, J. S. R. Alex, and N. Venkatesan, "Experimental evaluation of CNN architecture for speech recognition," in First International Conference on Sustainable Technologies for Computational Intelligence. Singapore: Springer, 2020, pp. 507-514. https: //doi.org/10.1007/978-981-15-0029-9_40

[23] Z. Huang, M. Dong, Q. Mao, and Y. Zhan, "Speech emotion recognition using CNN," in Proceedings of the 22nd ACM International Conference on Multimedia, 2014, Orlando, FL, pp. 801-804. https://doi.org/10.1145/2647868. 2654984

[24] N. N. Karnik, J. M. Mendel, and Q. Liang, “Type-2 fuzzy logic systems," IEEE Transactions on Fuzzy Systems, vol. 7, no. 6, pp. 643-658, 1999. https://doi.org/10.1109/91. 811231

[25] J. M. Mendel and R. B. John, “Type-2 fuzzy sets made simple," IEEE Transactions on Fuzzy Systems, vol. 10, no. 2, pp. 117-127, 2002. https://doi.org/10.1109/91.995115

[26] J. M. Mendel, "Uncertainty, fuzzy logic, and signal processing," Signal Processing, vol. 80, no. 6, pp. 913-933, 2000. https://doi.org/10.1016/S0165-1684(00)00011-6

[27] K. Simonyan and A. Zisserman, "Very deep convolutional networks for large-scale image recognition," in Proceedings of the 3rd International Conference on Learning Representations, San Diego, CA, 2015.

[28] T. L. Nguyen, S. Kavuri, and M. Lee, "A fuzzy convolutional neural network for text sentiment analysis," Journal of Intelligent \& Fuzzy Systems, vol. 35, no. 6, pp. 60256034, 2018. https://doi.org/10.3233/JIFS-169843

[29] S. Alizadeh and A. Fazel, "Convolutional neural networks for facial expression recognition," 2017, Available https: //arxiv.org/abs/1704.06756
[30] T. L. Nguyen, S. Kavuri, and M. Lee, "A multimodal convolutional neuro-fuzzy network for emotion understanding of movie clips," Neural Networks, vol. 118, pp. 208-219, 2019. https://doi.org/10.1016/j.neunet.2019.06.010

[31] S. J. Lee, T. Chen, L. Yu, and C. H. Lai, "Image classification based on the boost convolutional neural network," IEEE Access, vol. 6, pp. 12755-12768, 2018. https://doi.org/10.1109/ACCESS.2018.2796722

[32] E. A. Popko and I. A. Weinstein, "Fuzzy logic module of convolutional neural network for handwritten digits recognition," Journal of Physics: Conference Series, vol. 738, article no. 012123, 2016. https://doi.org/10.1088/ $1742-6596 / 738 / 1 / 012123$

[33] D.T. Mane and U. V. Kulkarni, "A novel fuzzy convolutional neural network for recognition of handwritten Marathi numerals," International Journal of High Performance Computing and Networking, vol. 15, no. 3-4, pp. 158-169, 2019. https://doi.org/10.1504/IJHPCN.2019 106094

[34] J. An, L. Fu, M. Hu, W. Chen, and J. Zhan, "A novel fuzzy-based convolutional neural network method to traffic flow prediction with uncertain traffic accident information," IEEE Access, vol. 7, pp. 20708-20722, 2019. https://doi.org/10.1109/ACCESS.2019.2896913

[35] U. Umoh, I. Umoeka, M. Ntekop, and E. Babalola, "Interval type-2 fuzzy neural networks for short-term electric load forecasting: a comparative study," International Journal on Soft Computing, vol. 9, no. 1, pp. 1-20, 2018. https://doi.org/10.5121/ijsc.2018.9101

[36] Y. LeCun, B. Boser, J. S. Denker, D. Henderson, R. E. Howard, W. Hubbard, and L. D. Jackel, "Backpropagation applied to handwritten zip code recognition," Neural Computation, vol. 1, no. 4, pp. 541-551, 1989. https://doi.org/10.1162/neco.1989.1.4.541

[37] J. M. Keller and D. J. Hunt, "Incorporating fuzzy membership functions into the perceptron algorithm," IEEE Transactions on Pattern Analysis and Machine Intelligence, vol. 7, no. 6, pp. 693-699, 1985. https://doi.org/10. 1109/TPAMI.1985.4767725

[38] S. R. Price, S. R. Price, and D. T. Anderson, "Introducing fuzzy layers for deep learning," in Proceedings of 2019 
IEEE International Conference on Fuzzy Systems (FUZZIEEE), New Orleans, LA, 2019, pp. 1-6. https://doi.org/ 10.1109/FUZZ-IEEE.2019.8858790

[39] A. Beke and T. Kumbasar, "Interval Type-2 fuzzy systems as deep neural network activation functions," in Proceedings of the 11th Conference of the European Society for Fuzzy Logic and Technology (EUSFLAT), Prague, Czech Republic, 2019, pp. 267-273. https://dx.doi.org/10.2991/ eusflat-19.2019.39

[40] V. V. Borisov, V. V. Kruglov, and A. S. Fedulov, Nechetkie modeli i seti [Fuzzy Models and Nets]. Moscow, Russia: Gorjachaja linija-Telekom, 2012.

[41] R. Fuller, Neural Fuzzy Systems. Turku, Finland: Abo Akademi University, 1995

[42] Q. Liang and J. M. Mendel, "Interval type-2 fuzzy logic systems: theory and design," IEEE Transactions on Fuzzy Systems, vol. 8, no. 5, pp. 535-550, 2000. https://doi.org/ $10.1109 / 91.873577$

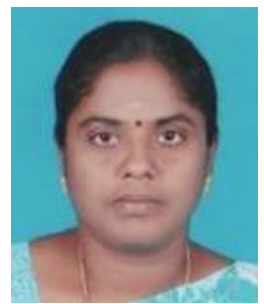

P. Murugeswari received the M.Tech degree in Computer Science and Information Technology from MS University, Tamilnadu, India in 2004 and the Ph.D. degree in Information and Communication Engineering from Anna University, Chennai, Tamilnadu, India in in 2014. She is a Professor of Computer Science and Engineering, Karpagam College of Engineering, Tamilnadu, Coimbatore, Tamilnadu, India since 2004. She received research fund from CSIR to conduct seminar, workshop and conference. She research has been published widely, more than 14 paper in reputed national/international journals and she has been invited to lecture on image processing and fuzzy logic Her research interest spans the domains of Image processing, Fuzzy logic, Neural Networks, Artificial Intelligence, Machine Learning and Data Science. Her research involves development of algorithm on Type-2 fuzzy logic based image classification using Deep learning concepts.

E-mail: pmurugeswarik7@gmail.com

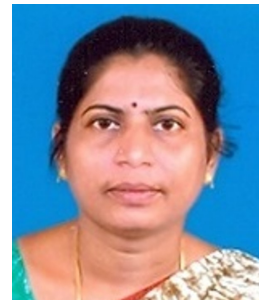

S.Vijayalakshmi received MCA degree from Madurai Kamaraj University, Madurai,Tamilnadu, India in 2000. and M.Phil degree in Computer Science and SET(State Eligibility Test ) in Computer Science and Applications from Mother Teresa University, Kodaikanal, Tamilnadu, and the Ph.D. degree in Computer Science from Bharathiar University, Coimbatore, Tamilnadu, India in in 2017. She is an Assistant Professor of Computer Applications Department, NMS S.Vellaichamy Nadar College, Madurai, Tamilnadu, India. She received a research fund from DRDO to conduct a seminar. Her research has been published widely, more than 10 papers in reputed national/international journals and she has been invited to lecture on Information Retrieval. Her research interest spans the domains of Text Mining, Artificial Intelligence, Neural Networks, Machine Learning and Data Science. Her research involves development of algorithms on Semi-Supervised Clustering using Deep learning concepts. E-mail: pandyviji@gmail.com 